\title{
Adverse events and disease flares after SARS-CoV-2 vaccination in patients with systemic lupus erythematosus
}

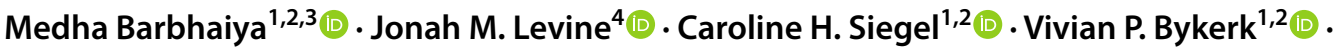 \\ Deanna Jannat-Khah ${ }^{1,2}$ (1) - Lisa A. Mandl ${ }^{1,2,3}$ (1)
}

Received: 5 August 2021 / Revised: 4 October 2021 / Accepted: 10 October 2021 / Published online: 30 October 2021 (c) International League of Associations for Rheumatology (ILAR) 2021

\section{Key Points}

- Over $91 \%$ of patients with SLE did not report disease flares postSARS-CoV-2 vaccination.

Dear Editor,

Vaccination against SARS-CoV-2 is particularly important for patients with systemic lupus erythematosus (SLE), who may be at increased risk of hospitalization from COVID-19 [1]. However, the most common reason for vaccine refusal is fear of SLE flare [2]. While SARS-CoV-2 mRNA vaccines could potentially induce interferon production and increase SLE activity [3], it is unclear if SARSCoV-2 vaccines are poorly tolerated in SLE.

In March 2021, we surveyed 466 SLE outpatients from a Rheumatology Division in New York City. SLE was defined using ICD-10 algorithms. Patients reported adverse events (AE) within 7 days of vaccination. Separately, patients reported "typical" disease flares within two weeks of vaccination. The study was approved by Hospital for Special Surgery Institutional Review Board.

183 patients with SLE responded (39.3\%); mean age 52.5 [SD 14.2] years; 94\% female; 65.6\%; White $15.9 \%$; Hispanic/Latinx. 136 (74.3\%) reported SARs-CoV-2 vaccination. Eighty-one (59.6\%) received Pfizer, 48 (39.3\%) received Moderna, (72/129 received both doses) and 4

Medha Barbhaiya

barbhaiyam@hss.edu

1 Division of Rheumatology, Hospital for Special Surgery, New York, NY, USA

2 Department of Medicine, Weill Cornell Medicine, New York, NY, USA

3 Department of Population Health Sciences, Weill Cornell Medicine, New York, NY, USA

4 Hospital for Special Surgery, New York, NY, USA
(2.9\%) received Janssen. Three vaccines manufacturers were unidentified.

One hundred patients (74\%) reported AEs: $61 \%$ after the first dose and $71 \%$ after the second. Most common were pain at injection site (54\%), fatigue (45\%), headache (36\%), sore shoulder (34\%), and muscle aches (26\%) (Supplement). No patients reported anaphylactic symptoms.

Eleven patients $(8.1 \%)$ reported flares (Table 1). These patients were older (59.8 [14.3] versus 54.2 [13.9] years) and more likely to be White (90.9\% versus $65.6 \%$ ). Only 1 patient who flared reported previous suspected/confirmed COVID-19 (9.1\% vs. $8 \%$ in the non-flare group). Flares occurred in $12.5 \%$ receiving Moderna $(N=6)$ and $6.2 \%$ receiving Pfizer $(N=5) ; 1 / 7$ patients who received both doses flared both times (Table 1). Of 12 total flares, 8 occurred after the first dose and 4 after the second. Medications to prevent or treat side effects were used by both flare and non-flare groups (Supplement). Most flares after the first dose were "mild" $(87.5 \%)$, whereas most after the second were "moderate" (75\%). Only one flare, after the 1 st dose, was severe and characterized by joint pain and brain fog, lasting 20 days (Table 1). Six of 12 flares started 1 day after vaccination, 4/12 started 4-7 days later, and none started $>7$ days later. Most flares resolved within 7 days; however, 3/12 lasted 8-21 days and 2/12 lasted $>21$ days.

We acknowledge possible misclassification of AEs as flares in the absence of confirmatory laboratory studies. However, we specifically asked patients to report symptoms concordant with their typical flares, separately from AEs. Although 100 patients reported AEs, only 11 reported a flare. This method for identifying flares is supported by data showing that SLE patients are reliable narrators of their disease experience and that self-reported SLE flares are associated with clinically meaningful outcomes [4, 5]. Given that the majority of patients reported AEs, whereas few reported flares, it does not appear that side effects alone explain our results. To prevent over interpretation of these data, we did not perform statistical testing. 


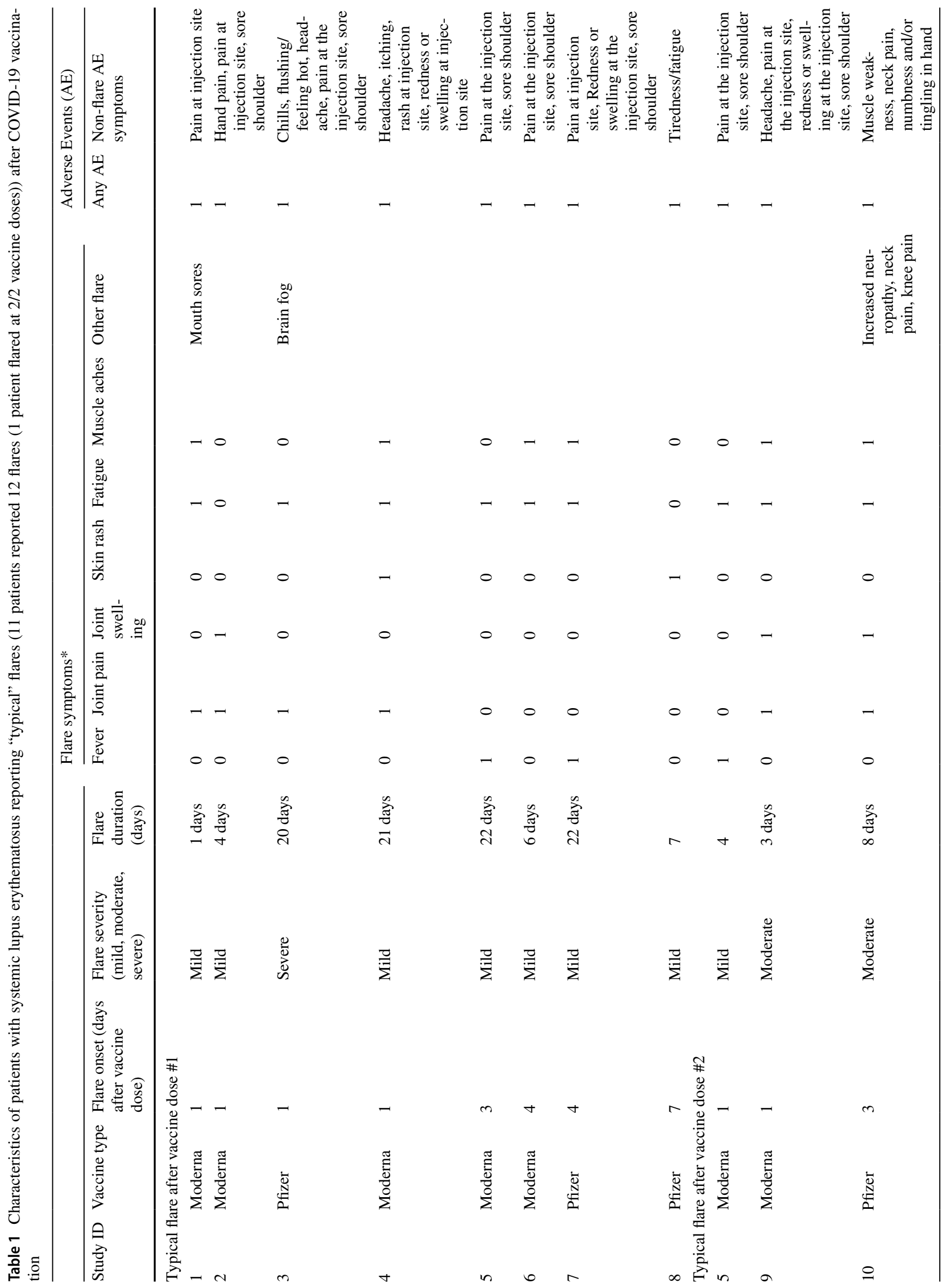


Our results are similar to a recent international study on outcomes after COVID-19 vaccination in patients with selfreported SLE [6]. Considering their US data for comparability, SLE patients in that study had a higher prevalence of flares $(7 / 60 ; 11.6 \%)$ and AEs $(66.7 \%$ after 1 st vaccine; $76.9 \%$ after 2 nd vaccine). Interestingly, they similarly found a higher prevalence of flares after the Moderna (8.8\%) than the Pfizer vaccine $(2.0 \%)$. Since validation of self-reported SLE can be as low as $11 \%$ [7], their different findings may reflect misclassified cases.

Our data suggest $>91 \%$ of SLE patients did not flare post-SARS-CoV-2 vaccination, and most flares were mild or moderate. Seventy-four percent reported a vaccine related AE, similar to the prevalence of AEs in the landmark Pfizer SARS-CoV-2 vaccine trial [8]. Whether factors such as vaccine manufacturer, or modifying immunosuppressive medications to enhance vaccine efficacy, independently predict flares in patients with SLE will need to be determined in larger cohorts. This information demonstrating that when flares occur, they are not severe, is reassuring. These data can help inform vaccine decision-making for patients with SLE.

Funding Dr. Barbhaiya is currently supported by the Rheumatology Research Foundation Investigator Award and the Barbara Volcker Center for Women and Rheumatic Diseases at Hospital for Special Surgery. Dr. Mandl received grant support from Regeneron Pharmaceuticals and is an Associate Editor at Annals of Internal Medicine. Dr. Siegel is supported by the National Center for Advancing Translational Sciences (NCATS) Grant \# UL1TR02384 of the Clinical and Translational Science Center at Weill Cornell Medical College. The funders had no role in study design, data collection, analysis, decision to publish, or preparation of the manuscript.

Data availability All data generated or analyzed during this study are included in this published article and its supplementary information files.

\section{Declarations}

Disclosures None.

\section{References}

1. Gianfrancesco M, Hyrich KL, Al-Adely S, Carmona L, Danila MI, Gossec L et al (2020) Characteristics associated with hospitalisation for COVID-19 in people with rheumatic disease: data from the COVID-19 Global Rheumatology Alliance physician-reported registry. Ann Rheum Dis 79(7):859-866. https://doi.org/10.1136/ annrheumdis-2020-217871

2. Chehab G, Richter JG, Brinks R, Fischer-Betz R, Winkler-Rohlfing B, Schneider M (2018) Vaccination coverage in systemic lupus erythematosus-a cross-sectional analysis of the German long-term 
study (LuLa cohort). Rheumatology (Oxford) 57(8):1439-1447. https://doi.org/10.1093/rheumatology/key120

3. Bengtsson AA, Ronnblom L (2017) Role of interferons in SLE. Best Pract Res Clin Rheumatol 31(3):415-428. https://doi.org/10. 1016/j.berh.2017.10.003

4. Katz P, Wan GJ, Daly P, Topf L, Connolly-Strong E, Bostic R et al (2020) Patient-reported flare frequency is associated with diminished quality of life and family role functioning in systemic lupus erythematosus. Qual Life Res 29(12):3251-3261. https:// doi.org/10.1007/s11136-020-02572-9

5. Katz P, Nelson WW, Daly RP, Topf L, Connolly-Strong E, Reed ML (2020) Patient-Reported Lupus Flare Symptoms Are Associated with Worsened Patient Outcomes and Increased Economic Burden. J Manag Care Spec Pharm 26(3):275-283. https://doi. org/10.18553/jmcp.2020.26.3.275

6. Felten R, Kawka L, Dubois M, Ugarte-Gil MF, Fuentes-Silva $\mathrm{Y}$, Piga $\mathrm{M}$ et al (2021) Tolerance of COVID-19 vaccination in patients with systemic lupus erythematosus: the international VACOLUP study. Lancet Rheumatol. https://doi.org/10.1016/ S2665-9913(21)00221-6

7. Walitt BT, Constantinescu F, Katz JD, Weinstein A, Wang H, Hernandez RK et al (2008) Validation of self-report of rheumatoid arthritis and systemic lupus erythematosus: The Women's Health Initiative. J Rheumatol 35(5):811-818

8. Polack FP, Thomas SJ, Kitchin N, Absalon J, Gurtman A, Lockhart S et al (2020) Safety and efficacy of the BNT162b2 mRNA Covid-19 vaccine. N Engl J Med 383(27):2603-2615. https://doi. org/10.1056/NEJMoa2034577

Publisher's note Springer Nature remains neutral with regard to jurisdictional claims in published maps and institutional affiliations. 